\title{
Preliminary Data on a SERS-Responsive Sensor Based on Metallic Nanostructures Functionalized by Aptamers Specific for Arsenic
}

\section{Domenica Musumeci ${ }^{1, a}{ }^{*}$, Daniela Montesarchio ${ }^{1, b}$, Elisa Scatena ${ }^{2, \mathrm{c}}$, Costantino Del Gaudio ${ }^{2, \mathrm{~d}}$, Fabio De Matteis ${ }^{3, \mathrm{e}}$, Roberto Francini ${ }^{3, \mathrm{f}}$, Mauro Casalboni, ${ }^{3,{ }^{*}}$}

${ }^{1}$ Dept. of Chemical Sciences, Federico II University of Napoli, via Cinthia 4, 80126 Napoli, Italy

${ }^{2}$ Fondazione E. Amaldi, via del Politecnico snc, 00133 Roma, Italy

${ }^{3}$ Dept. of Industrial Engineering, Tor Vergata University of Roma, via del Politecnico 1, 00133 Roma, Italy

\author{
adomenica.musumeci@unina.it, bdaniela.montesarchio@unina.it, \\ celisa.scatena@fondazioneamaldi.it, ${ }^{d}$ costantino.delgaudio@fondazioneamaldi.it, \\ edematteis@roma2.infn.it, ${ }^{\mathrm{f}}$ francini@roma2.infn.it, ${ }^{9}$ casalboni@uniroma2.it \\ * corresponding authors
}

Keywords: Arsenic Detection, SERS, Oligonucleotide Aptamers

\begin{abstract}
Arsenic, in the form of arsenate $\left(\mathrm{As}^{\mathrm{V}}\right)$ and arsenite (As $\left.{ }^{\mathrm{III}}\right)$, is a toxic carcinogen widely distributed in aqueous environments in many parts of the world. Efficient arsenic sensors in terms of sensitivity, selectivity, speed and portability are urgently needed. The present research was focused on the development of a gold nanostructured sensor, functionalized with organic molecules able to selectively bind arsenic, for SERS detection. As organic molecule, we here selected oligonucleotide aptamers specific for arsenic recognition relying on previous studies on a 100-mer arsenic-binding DNA aptamer (Ars3), selected for its highest affinity and specificity to arsenate and arsenite. The aptamer Ars3 was previously used as such in its whole sequence and no attempt to optimize it, in terms of size and sensor efficiency, or to unravel its binding mechanism with arsenic has been carried out. Furthermore, even if in the previously proposed Ars3-based sensors a low detection limit for As was achieved, the assembly of the probe and the detection methodologies were in most cases very complex and not suitable for the development of portable in situ devices. Thus, in order to optimize Ars3 and investigate its interaction with arsenic, we here designed shorter DNA sequences cutting the 3' and 5' ends of the parent aptamer, and carried out spectroscopic and electrophoretic analysis together with arsenic-binding assays by using a suitably functionalized affinity resin. In addition, a specific SERS-responsive system with the Ars3 parent aptamer was considered in view of its application with the modified DNA sequences here proposed. Collected findings highlighted that the parent aptamer did not bind arsenic with high affinity. This was also in agreement with very recent results, published concomitantly with our studies, which stated that Ars3 was not able to bind As and that all the positive binding data using Ars3 were due to the use of gold supports (SPR chips or gold nanoparticles) which tightly bound arsenic. Based on these results, no arsenic-binding DNA aptamers are currently known, thus underlining the need for actual arsenic binding aptamers to be implemented for SERS sensors.
\end{abstract}

\footnotetext{
(c) (1) Content from this work may be used under the terms of the Creative Commons Attribution 3.0 license. Any further distribution of this work must maintain attribution to the author(s) and the title of the work, journal citation and DOI. Published under license by Materials Research Forum LLC.
} 


\section{Introduction}

Arsenic poisoning is caused by elevated levels of arsenic in the body. The dominant basis of arsenic poisoning is from ground water that naturally contains high concentration of arsenic [1]. Chronic ingestion or long-term exposure to arsenic can cause a variety of cancer forms, skin lesions, and cardiovascular diseases [1]. Elevated concentrations of arsenic in ground water have been found in many parts of the world and the maximum tolerable value of arsenic in drinkable water, in agreement with the World Health Organization, is $10 \mu \mathrm{g} / \mathrm{L} \mathrm{(ppb)} \mathrm{[2].} \mathrm{It} \mathrm{is} \mathrm{generally}$ recognized that inorganic arsenic is more toxic than organic one, and among inorganic arsenic, arsenic in oxidation state +3 (i.e., arsenite, derived from arsenous acid) is more toxic than that in the oxidation state +5 (i.e., arsenate, derived from arsenic acid) [1].

Currently, the most used analytical methodologies for the detection of arsenic at very low concentrations are Atomic Fluorescence Spectroscopy (AFS), Atomic Absorption Spectroscopy (AAS) and Inductively Coupled Plasma Mass Spectrometry (ICP-MS) [1],[3],[4]. These techniques are very efficient, but not suitable for in situ analysis, expensive, time consuming, and require chemical and physical pre-treatment of the samples.

Therefore, it is essential not only to develop sensitive and selective analytical techniques for the detection of arsenic even in traces in the environment, but also to construct efficient arsenicspecific sensors in terms of speed and portability, especially for developing countries with highrisk contaminated soils. In this context, Surface-Enhanced Raman Scattering (SERS) guarantees high sensitivity and allows engineering tools of extremely reduced size. SERS can accomplish the speciation analysis for species with different oxidation states, with no need for any complex preparation of the sample, and has thus emerged as an extremely promising solution for in situ detection of arsenic in the field, particularly when coupled with portable/handheld Raman spectrometers [1],[5].

The present research was focused on the realization of a gold nanostructured sensor for SERS detection, functionalized with organic molecules able to selectively bind arsenic. As organic molecules, we here selected oligonucleotide aptamers specific for arsenic recognition.

Aptamers are DNA/RNA oligonucleotides (ON) able to recognize with high affinity and selectivity a specific target based on their peculiar three-dimensional structures [6]. Aptamers can be selected from huge libraries of molecules containing randomly generated sequences, and specifically bind different targets, from proteins, viruses or whole cells to small molecules or ions [6],[7].

About 10 years ago a Korean group identified a 100-mer DNA aptamer (named Ars3) having high affinity and specificity for arsenate $\left(\mathrm{As}^{\mathrm{V}}\right)$ and arsenite $\left(\mathrm{As}^{\mathrm{III}}\right)$, with dissociation constants in the low $\mathrm{nM}$ range [8]. This arsenic-binding aptamer was selected from a random DNA library of 100-mer oligonucleotides which contained two constant regions at their ends as well as a $40 \mathrm{nt}$ random region in the middle: ${ }^{5^{\prime}}$ GGT AAT ACG ACT CAC TAT AGG GAG ATA CCA GCT TAT TCA ATT-N 40 -AGA TAG TAA GTG CAA TCT ${ }^{3}$.

The aptamers were selected using affinity chromatography-based SELEX (Systematic Evolution of Ligands by EXponential enrichment), obtained by immobilizing arsenic on an agarose resin. Quantitative analyses of the aptamer candidates by SPR revealed Ars3 as the aptamer with the highest affinity to arsenate and arsenite. The specific affinity interactions of the aptamer to arsenic were verified against other heavy metals as negative controls, confirming its high specificity [8].

From this first work, Ars3 has been explored also in subsequent researches on arsenic sensors. In all cases, the aptamer was used as such, that is containing the random part plus the two flanking constant regions (which were generally removed) required for the PCR (polymerase chain reaction) step of the SELEX, and no attempt to optimize it, in terms of size and sensor- 
efficiency, or to elucidate its binding mechanism with arsenic has been ever described [9-17]. Furthermore, even if the detection limit for As sensors previously reported exploiting Ars3 was generally low, the assembly of the probe and the detection methodologies were very complex and not suitable for the development of portable in situ devices.

Thus, in order to optimize Ars3 and further analyse its interaction with arsenic, we designed shorter DNA sequences, cutting in various way the 3' and 5' ends of the parent aptamer, and carried out spectroscopic and electrophoretic analyses together with arsenic-binding assays by using a suitably functionalized affinity resin. Moreover, a SERS-responsive system to Ars3 was ad hoc implemented in view of its application with the modified DNA sequences here proposed.

\section{Results and Discussion}

The detection procedure and the arsenic-binding aptamer Ars3 optimization were both addressed, designing a set of shorter oligonucleotide sequences starting from the original 100-mer, cutting its constant parts in various ways but leaving unaltered the central core of the sequence, which should contain the residues necessary for the specific recognition.

From the analysis of all the designed sequences using $m$-fold program we selected three sequences (Ars40, Ars58 e Ars64, Fig. 1) potentially able to form hairpin-loop structures with good stability as the parent aptamer.

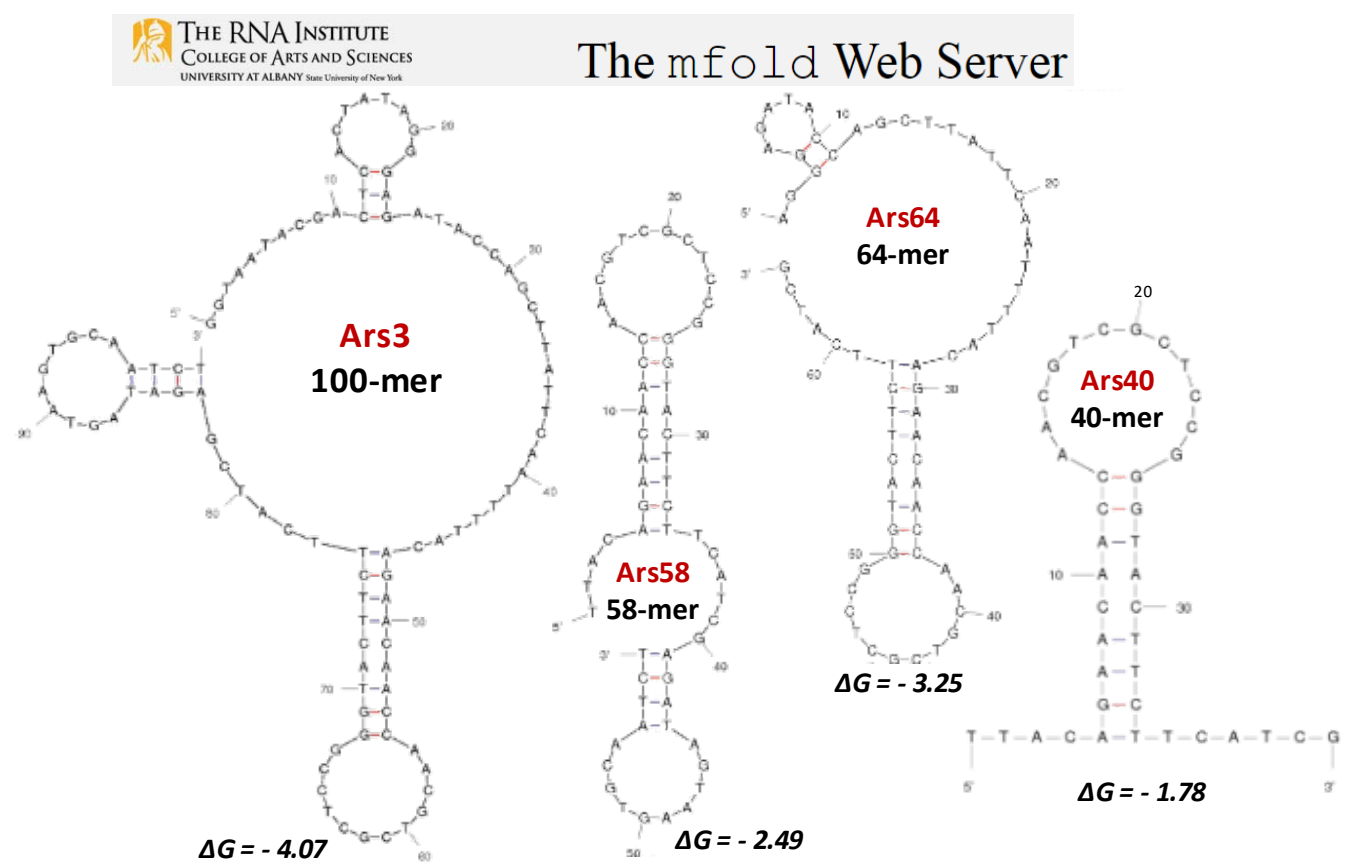

Figure 1: Folding prediction of Ars3 and the derived shortened sequences obtained using the mfold program (settings: $150 \mathrm{mM} \mathrm{NaCl}, 5 \mathrm{mM} \mathrm{MgCl}_{2}$; folding temperature, $25^{\circ} \mathrm{C}$ ).

Then, once obtained the set of the selected sequences, we analysed them by circular dichroism (CD) spectroscopy in two different buffers: the selection buffer originally used for Ars3 aptamer (named SB, $50 \mathrm{mM}$ Hepes, $150 \mathrm{mM} \mathrm{NaCl}, 0.5 \mathrm{mM} \mathrm{MgCl}_{2}, 0.5 \mathrm{mM} \mathrm{CaCl}_{2}, \mathrm{pH} 7.5$ ) and PBS (phosphate buffered saline, $137 \mathrm{mM} \mathrm{NaCl}, 2.7 \mathrm{mM} \mathrm{KCl}, 10 \mathrm{mM} \mathrm{Na}_{2} \mathrm{HPO}_{4}, 1.8 \mathrm{mM} \mathrm{KH}_{2} \mathrm{PO}_{4}, \mathrm{pH}$ 7.4). All the sequences showed in both buffers almost the same conformational behaviour, with quite similar bands in their CD spectra, evidencing the formation of B-DNA duplex tracts probably due to hairpin-loop structures (Fig. 2a). CD-melting curves showed the highest melting temperature $\left(\mathrm{T}_{\mathrm{m}}\right)$ for the 100 -mer $\mathrm{ON}$ and the lowest $\mathrm{T}_{\mathrm{m}}$ value for the 40 -mer (Fig. $2 \mathrm{~b}$ ). All the 
sequences were analysed in both annealed (A) and not annealed (NA) form in order to explore both the thermodynamically and kinetically favoured conformations, respectively. The CD spectra of both the annealed and not-annealed samples were in all cases quite superimposable. This result proved that the kinetically more stable conformations were essentially similar to the thermodynamically favoured ones for each ON.
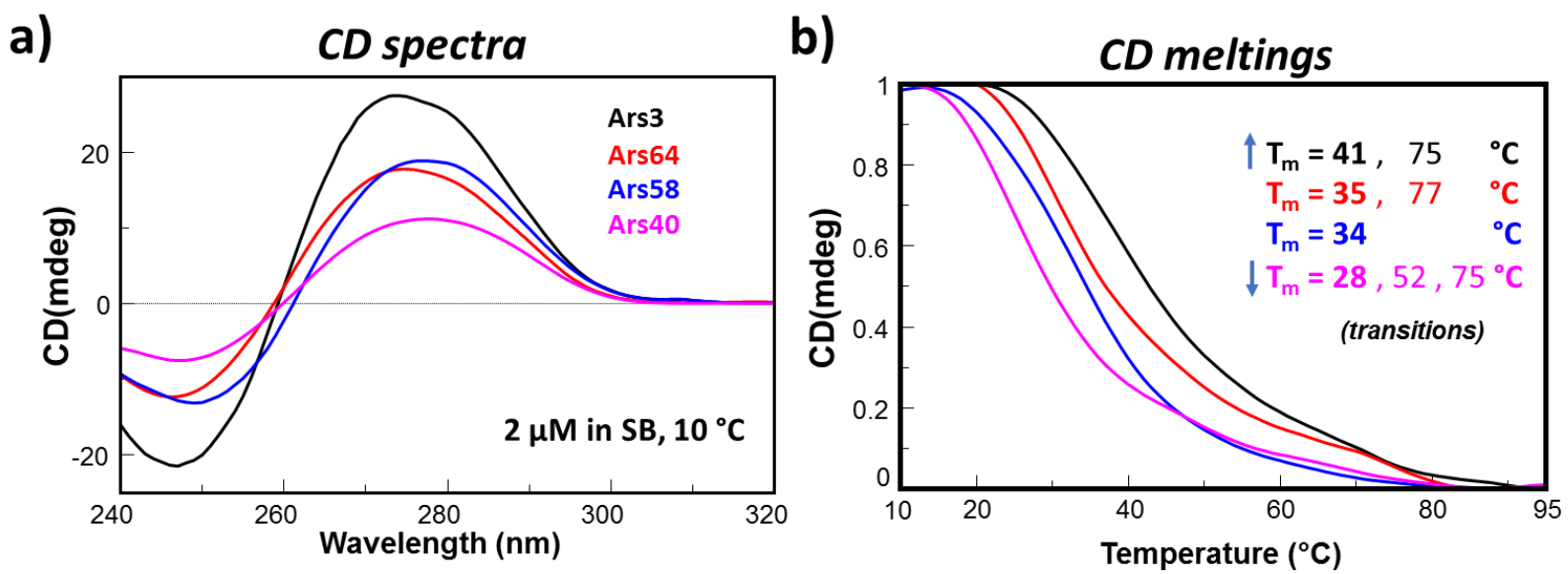

Figure 2: a) Overlapped CD spectra of the four not-annealed ONs in SB at $2 \mu \mathrm{M}$ conc. at $10^{\circ} \mathrm{C}$; b) normalized CD-melting curves of the solutions in a).

Analysis of the electrophoretic mobility of the annealed and not annealed forms of the four investigated sequences through native PolyAcrilamide Gel Electrophoresis (PAGE) excluded the formation of bimolecular or higher-order structures since a single band for each oligonucleotide was observed. Thus, all the sequences formed exclusively monomolecular species in the selected buffer and concentration conditions (Fig. 3).

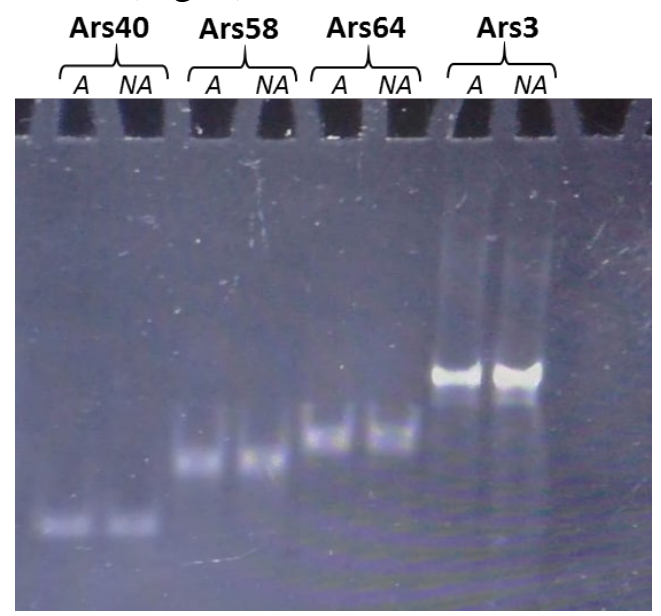

Figure 3: Electrophoretic mobility analysis of the annealed and not annealed forms of the investigated sequences at $2 \mu M$ conc. in SB. Native $7 \%$ polyacrylamide gel electrophoresis (PAGE) with TBE $1 x$ as running buffer.

In parallel, in order to design the arsenic sensor and set up the system for arsenic detection, we preliminarily attached the parent aptamer (that is the 100-mer Ars3) on a substrate suitable for SERS analysis. This substrate was based on highly packed gold-coated nanopillars structured on a silicon wafer. The aptamer was modified at its 5' end with a C6-linker carrying a thiol group useful for the immobilization on the nanopillars via Au-S covalent bonds (Fig. 4). In brief, 
after the deposition of the target solution on the functionalized substrate, the wetted surface was allowed to dry. Due to the surface tension between pillars during liquid evaporation, the nanopillars, leaning toward each other, formed micro-sized clusters with highly localized plasmonic field around the contact region (the so-called hotspots). When the analyte was trapped by an aptamer within a hotspot, the Raman signal was highly enhanced.
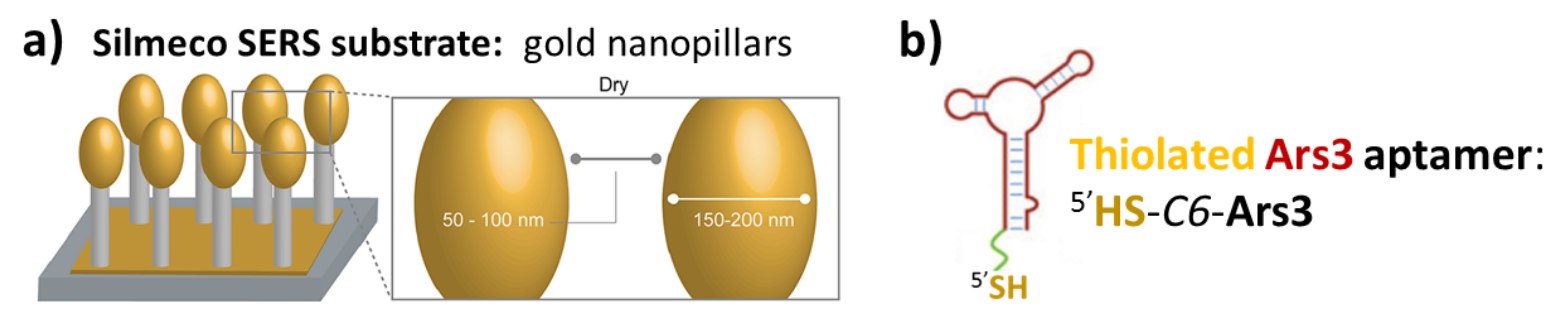

Dimensions: $4 \times 4 \mathrm{~mm}$; Sensitivity: $\mathrm{ppm}$ to $\mathrm{ppb}$

Figure 4: Schematic representations of a) a Silmeco substrate for SERS analysis and b) the 5'thiolated Ars3 aptamer.

Thus, we prepared two substrates: 1) a reference substrate containing Ars3 and the 6mercapto-1-hexanol (MCH) as passivating agent, and 2) another substrate containing also As ${ }^{\text {III }}$ added as analyte. In a preliminary assay, we analysed in label-free mode the two prepared substrates and evidenced some remarkable differences in their SERS spectra (Fig. 5).

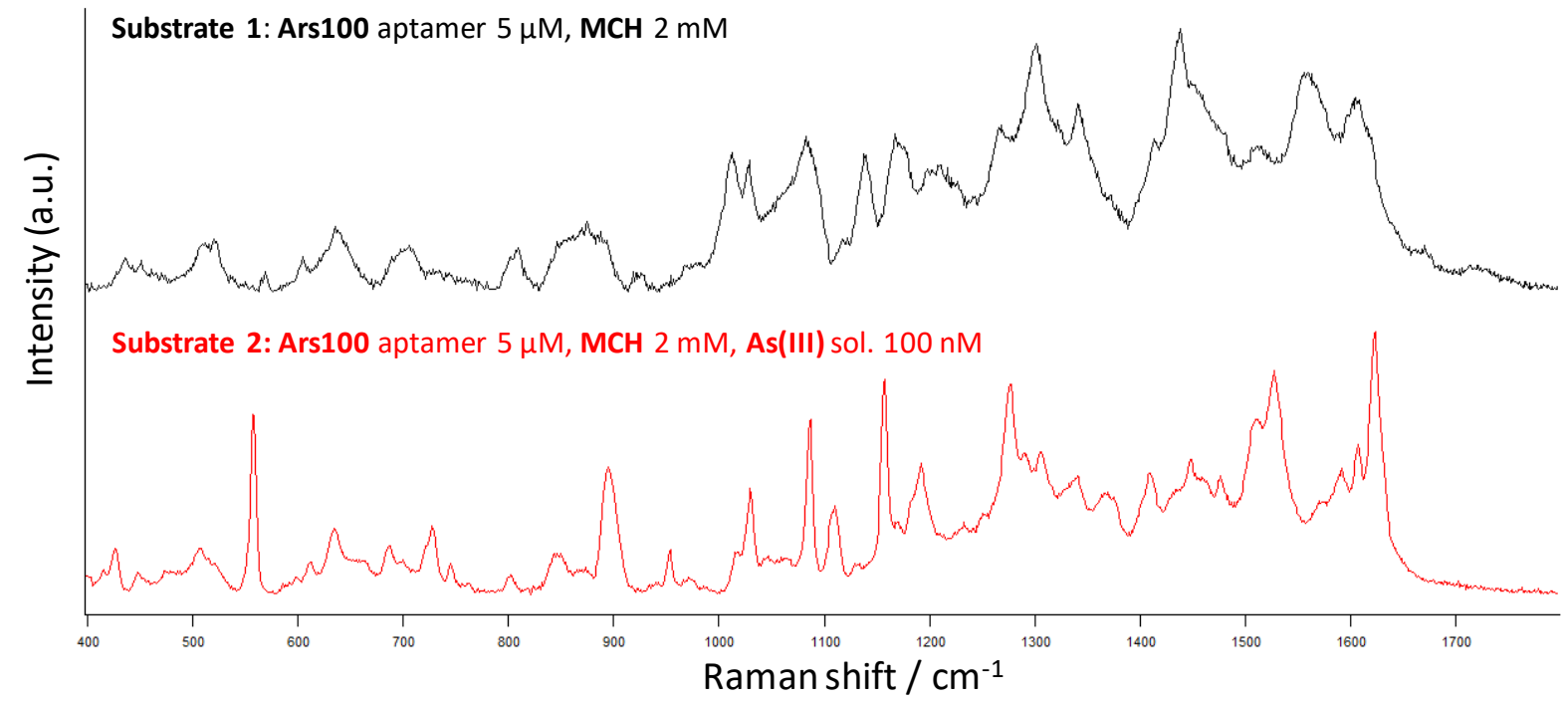

Figure 5: Preliminary SERS data for As ${ }^{I I I}$ detection by Ars3-functionalized Silmeco substrates.

We then performed CD-titrations in SB or PBS solutions of the original aptamer and the shorter analysed sequences (in both annealed and not-annealed form) with both $\mathrm{As}^{\mathrm{III}}$ and $\mathrm{As}^{\mathrm{V}}$ salts in order to investigate the possible conformational changes due to arsenic binding and thus measure the affinity and stoichiometry of these interactions.

In all cases the CD spectra of all the tested ONs remained unchanged upon As titration (data not shown): thus, even if the binding with arsenite and arsenate occurred, this did not affect the conformation of the ONs, including the parent aptamer Ars3. This result was in net disagreement with various works reporting that marked CD changes occurred after arsenic addition to Ars3 [9],[11],[15],[16]. 
Furthermore, also the thermodynamic stability of the structured ONs (monitored through CDmelting experiments) did not show variations in the presence of arsenic: indeed, the melting temperatures $\left(\mathrm{T}_{\mathrm{m}}\right)$ for all the investigated systems (ON-As) remained almost unchanged, at least in the selected concentration and buffer conditions, with respect to the solutions of the free ONs. Therefore, from CD experiments we were not able to measure the affinity of Ars3 and of the other sequences for arsenic since no change in the CD spectra derived from arsenic addition to the ONs was evidenced: this behaviour however was not unusual since it was found also for other DNA binders, especially those providing groove interactions with duplex or G-quadruplex DNA structures.

Subsequently, in order to further check the binding affinity of Ars3 for arsenic, using a technique alternative to SPR or CD, we decided to exploit affinity chromatography taking inspiration from the first work on Ars3. We thus anchored arsenic(III) (in the form of its salt $\mathrm{NaAsO}_{2}$ ) on an agarose resin (Fig. 6a). First, the resin, containing activated carboxylic groups was covalently coupled with 1,8-diaminooctane and its incorporation was qualitatively evaluated through ninhydrin test (few mg of resin containing free amino groups, assumed a strong blueviolet colour reacting at high temperature with ninhydrin; on the contrary the control amine-free resin remained colourless, Fig. 6b). Then, arsenite was linked exploiting electrostatic interactions between its negative charge and the ammonium moiety on the resin, using a protocol optimized for the $\mathrm{pH}$ and concentration of the arsenite solution (Fig. 10a).

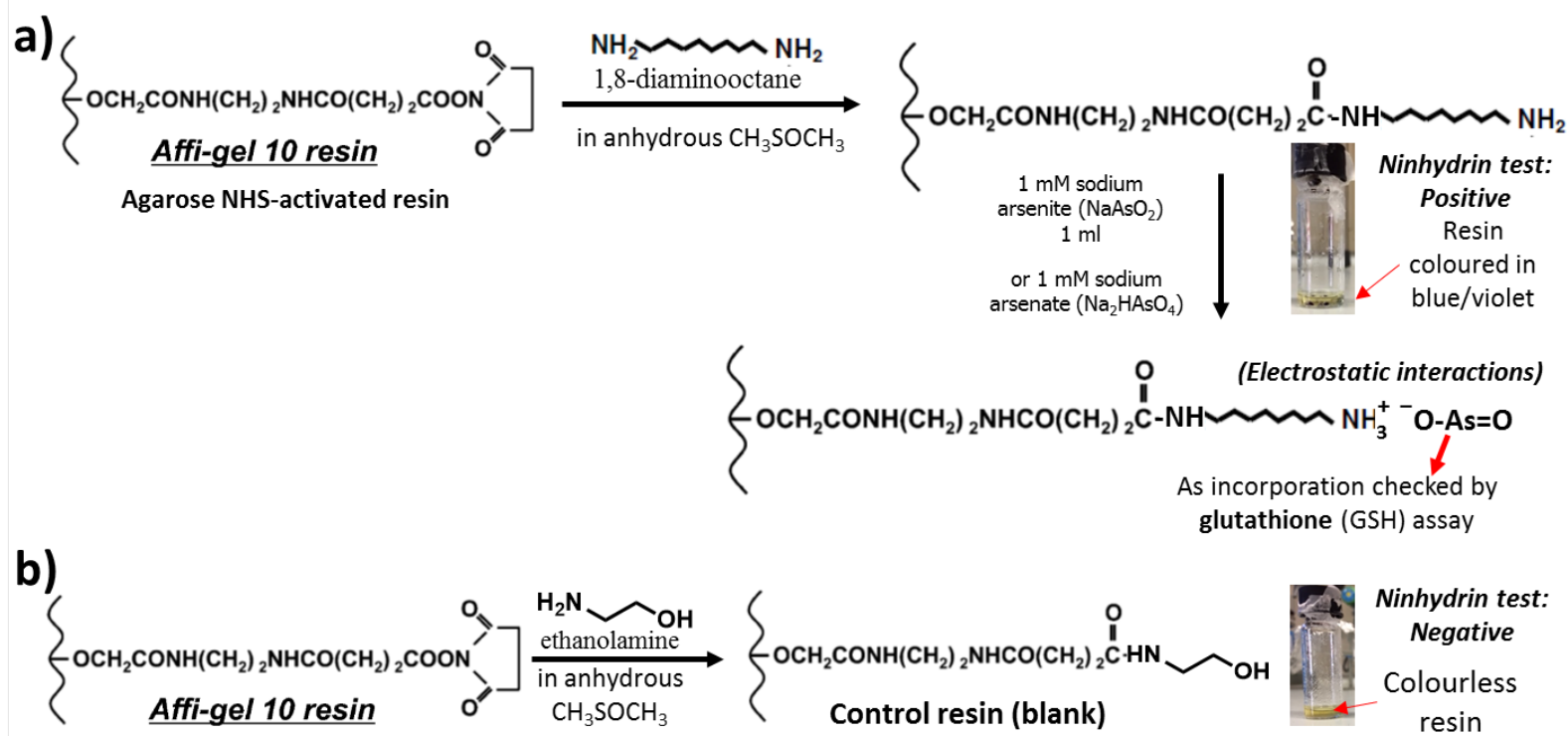

Figure 6: Schematic representation of the preparation of the As-funcionalized (a) and control (b) resins.

Arsenic incorporation on the resin was evaluated through glutathione (GSH) assay, ad hoc adapted for the system we previously assembled in solid phase. This assay is based on the formation of a stable complex between the thiol group of the GSH and arsenic ${ }^{\text {III }}$ which shows a characteristic UV-band at $290 \mathrm{~nm}$ [18].

Thus, after incubation of the amine resin with arsenite solution, first, the excess of arsenic was washed away, and then the As ${ }^{\mathrm{III}}$ bound to the resin was subsequently released using a $20 \mathrm{mM}$ GSH solution and quantified by UV (Fig. 7). 
Subsequently, we planned to set up the As ${ }^{\text {III }}$ affinity assay for Ars3 and the modified sequences. The solutions of the four oligonucleotides (both in annealed and not-annealed form) in PBS or SB were loaded on the resin separately and the eluted fractions were collected, including various washings, and analysed by UV measurements. From the difference in the absorption of all the ON solutions (band at $260 \mathrm{~nm}$ ) before and after loading them on the resin, we calculated the percentage content of ON captured by the resin and thus the affinity of arsenic for each sequence, including the parent Ars3 aptamer. The unspecific absorption on the blank resin, which however was minimal, was also considered and subtracted.
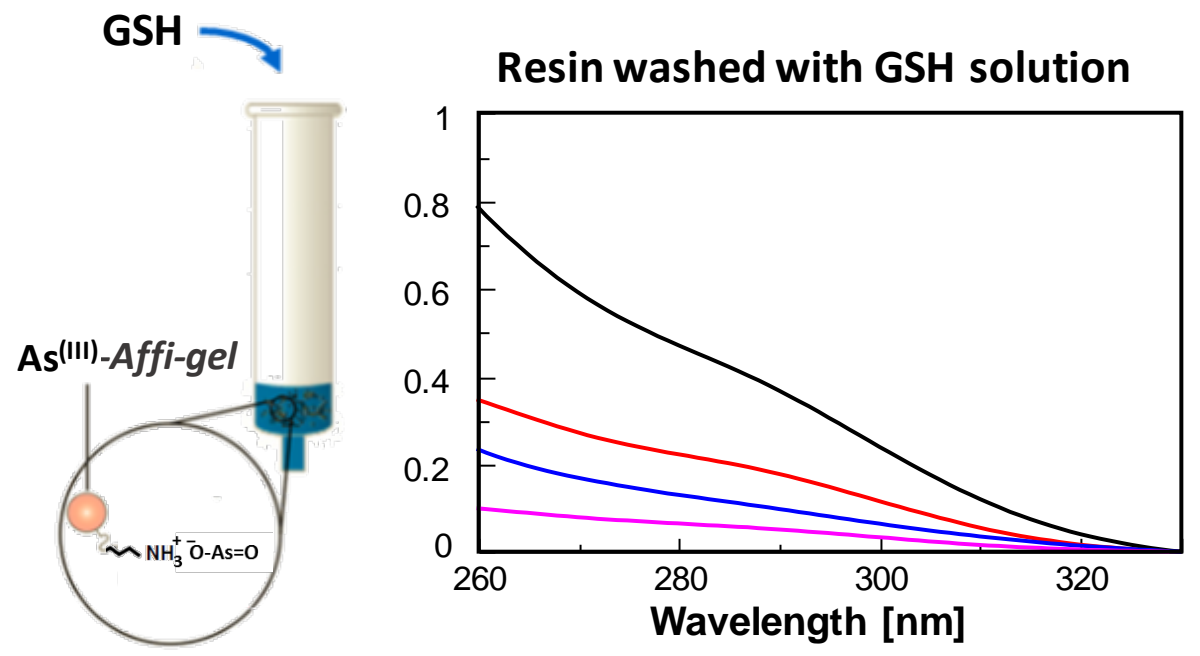

Figure 7: Schematic representation of the GSH assay on the As-functionalized Affigel resin and overlapped UV spectra of the fraction eluted from the resin after GSH solution washings.

Unfortunately, the binding assay did not give the expected results (neither for the reference aptamer Ars3) and always provided not reproducible data. Thus, after a number of experiments, performed changing various parameters of the binding conditions (concentrations, temperature, incubation time, etc.), we concluded that Ars3 did not show the expected nM affinity for arsenite, and that $\mathrm{As}^{\mathrm{III}}$ was washed away from the resin in various amount together with the oligonucleotide solutions or the buffer, as verified by the GSH assay.

Probably, the electrostatic interactions were not sufficient to keep the arsenite stably linked on the resin as stated in ref. [8] relatively to the SPR binding assays. Thus, we found many contradictory results with respect to the first work on the arsenic binding aptamer Ars3 [8]. Indeed, in parallel to our experiments two very recent works demonstrated the high affinity of arsenite for gold and the false positive generated from the interactions of aptamer and As ${ }^{\text {III }}$ on gold surface-involving assays [19-20]. Particularly, in ref [20], the ability of aptamer Ars3 to bind arsenite and arsenate was confuted by accurate ITC (Isothermal Titration Calorimetry) measurements. Thus, our difficulty to reproduce the binding assay of Ars3 with As ${ }^{\mathrm{III}}$ on an agarose affinity resin (instead of the original gold-based SPR assay) was actually due to the absence of affinity of the aptamer for the arsenic target, as well as to the partial detachment from the resin of electrostatically-immobilized arsenic after repeated washings of the resin with buffered solutions.

Thus, on the basis of the recent literature reports [19-20] and of our scientific results, we can definitely exclude the DNA aptamer Ars3 as a candidate for specifically bind arsenic in a SERSbased sensor. Our studies are now directed towards peptide aptamers, specifically focussing on 
high cysteine-content peptides developed ad hoc for arsenic(III) sensing and using silver substrates for SERS detection instead of gold.

\section{Experimental part}

The DNA sequences used in this study are:

Ars-3: ' ${ }^{\prime}$ GGTAATACGACTCACTATAGGGAGATACCAGCTTATTCAATTTTACAGAACA ACCAACGTCGCTCCGGGTACTTCTTCATCGAGATAGTAAGTGCAATCT ${ }^{3}$ '(100mer).

Ars64: ${ }^{5}$ AGGGAGATACCAGCTTATTCAATTTTACAGAACAACCAACGTCGCTCC GGGTACTTCTTCATCG ${ }^{3}$ ' (64-mer, excluding 18 bases both at 5' and 3' ends); Ars58: $\quad$ 5'TTACAGAACAACCAACGTCGCTCCGGGTACTTCTTCATCGAGATAGT AAGT GCAATCT ${ }^{3}$ (58-mer, excluding the 42-base constant sequence at 5' end);

Ars40: '5'TACAGAACAACCAACGTCGCTCCGGGTACTTCTTCATCG ${ }^{3}$ (40 mer, excluding both the constant regions of 42 and 18 bases at 5' and 3' ends, respectively).

All the $\mathrm{ON}$ sequences were purchased by biomers.net $\mathrm{GmbH}$ in lyophilized and desalted form. The annealed (A) aptamer solutions were prepared by diluting the stock aptamer solution in the selected buffer, heating it for $5 \mathrm{~min}$ at $95{ }^{\circ} \mathrm{C}$ and then allowing it to slowly cool to r.t. In turn, the not annealed (NA) aptamer solutions were prepared by diluting the stock aptamer solution in $\mathrm{H}_{2} \mathrm{O}$ at r.t. in the selected buffer. $\mathrm{CD}$ melting experiments were performed by following the CD-band maximum of each ON upon increasing the temperature (scan rate: 1 $\left.{ }^{\circ} \mathrm{C} / \mathrm{min}\right)$.

Electrophoresis analysis: run, $89 \mathrm{~V} 1$ h,30 min; staining, Gel green; visualization, UV ChemiDoc transilluminator.

SERS analysis: excitation source, $785 \mathrm{~nm}$ He-Ne laser; grating, 1200; objective: 50x; exp. Time 10s.

CD titration: ON solutions ( $2 \mu \mathrm{M}$ in $\mathrm{A}$ and $\mathrm{NA}$ form) in PBS and SB were titrated with $\mathrm{NaAsO}_{2}$ and $\mathrm{NaAsO}_{4}$ (conc. from $2 \mu \mathrm{M}$ to $1 \mathrm{mM}$ ) and CD spectra were registered at different time periods ( 3 min, 30 min, 2 h, 24 h).

\section{Summary}

The present research was focused on the development of a gold nanostructured sensor, functionalized with DNA aptamers able to selectively bind arsenic, for SERS detection. In order to optimize the previously reported aptamer Ars3 (a 100-mer DNA) and investigate its interaction with arsenic, we here designed shorter DNA sequences cutting the 3' and 5' ends of the parent aptamer and carried out spectroscopic and electrophoretic analysis together with arsenic-binding assays by using a suitably functionalized affinity resin. In addition, a specific SERS-responsive system with the Ars3 parent aptamer was considered in view of its application with the modified DNA sequences here proposed. Collected findings highlighted that the parent aptamer did not bind arsenic with high affinity, in agreement with very recent results, published concomitantly with our studies, which stated that Ars3 was not able to bind As. Based on these results, no DNA aptamers specifically recognizing arsenite/arsenate are currently known, thus underlining the need for actual arsenic binding aptamers to be implemented for SERS sensors.

\section{Acknowledgements}

This research was funded by Regione Lazio, through Progetto di ricerca 85-2017-15173 according to L.R. 13-08. Progetto NARAS. 


\section{References}

[1] M. Bissen, F.H. Frimmel, Arsenic: A review. Part I: Occurrence, toxicity, speciation, mobility, Acta. Hydrochim. Hydrobiol. 31 (2003) 9-18. https://doi.org/10.1002/aheh.200390025

[2] Information on https://www.who.int/news-room/fact-sheets/detail/arsenic

[3] N. Yogarajah, S.S.H. Tsai, Detection of trace arsenic in drinking water: challenges and opportunities for microfluidics, Environ. Sci.: Water Res. Technol. 1 (2015) 426-447. https://doi.org/10.1039/C5EW00099H

[4] A.K Farzana, Z. Chen, L. Smith, D. Davey, R. Naidu, Speciation of arsenic in ground water samples: A comparative study of CE-UV, HG-AAS and LC-ICP-MS, Talanta 68 (2005) 406-15. https://doi.org/10.1016/j.talanta.2005.09.011

[5] J. Hao, M.J. Han, S. Han, X. Meng, T.L. Su, Q.K. Wang, SERS detection of arsenic in water: A review, J. Environ. Sci. (China) 36 (2015) 152-62. https://doi.org/10.1016/j.jes.2015.05.013

[6] H. Kaur, J.G. Bruno, A. Kumar, T.K. Sharma, Aptamers in the Therapeutics and Diagnostics Pipelines, Theranostics, 8 (2018) 4016-4032. https://doi.org/10.7150/thno.25958

[7] C. Platella, C. Riccardi, D. Montesarchio, G.N. Roviello, D. Musumeci, G-quadruplex-based aptamers against protein targets in therapy and diagnostics, Biochim. Biophys. Acta Gen. Subj. 2017;1861(5 Pt B):1429-1447. https://doi.org/10.1016/j.bbagen.2016.11.027

[8] Kim M, Um HJ, Bang S, Lee SH, Oh SJ, Han JH, Kim KW, Min J and Kim YH. Arsenic Removal from Vietnamese Groundwater Using the Arsenic-Binding DNA Aptamer. Environ. Sci. Technol. 2009, 43: 9335-9340. https://doi.org/10.1021/es902407g

[9] Wu Y, Liu L, Zhan S, Wang F, Zhou P. Ultrasensitive aptamer biosensor for arsenic(III) detection in aqueous solution based on surfactant-induced aggregation of gold nanoparticles. Analyst. 2012, 137: 4171-8. https://doi.org/10.1039/c2an35711a

[10] Wu Y, Zhan S, Wang F, He L, Zhi W, Zhou P. Cationic polymers and aptamers mediated aggregation of gold nanoparticles for the colorimetric detection of arsenic(III) in aqueous solution. Chem Commun (Camb). 2012 May 11;48(37):4459-61.

https://doi.org/10.1039/c2cc30384a

[11] Wu Y, Zhan S, Xing H, He L, Xu L, Zhou P. Nanoparticles assembled by aptamers and crystal violet for arsenic(III) detection in aqueous solution based on a resonance Rayleigh scattering spectral assay. Nanoscale. 2012 Nov 7;4(21):6841-9. https://doi.org/10.1039/c2nr31418e

[12] S. Zhan, M. Yu, J. Lv, L. Wang, P. Zhou, Colorimetric Detection of Trace Arsenic(III) in Aqueous Solution Using Arsenic Aptamer and Gold Nanoparticles, Aust. J. Chem. 67 (2014) 813-818. https://doi.org/10.1071/CH13512

[13] F. Divsar, K. Habibzadeh, S. Shariati, M. Shahriarinourc, Aptamer conjugated silver nanoparticles for the colorimetric detection of arsenic ions using response surface methodology, Anal. Methods. 7 (2015) 4568-4576. https://doi.org/10.1039/C4AY02914C

[14] L. Song, K. Mao, X. Zhou, J. Hu, A novel biosensor based on Au@Ag core-shell nanoparticles for SERS detection of arsenic (III), Talanta 146 (2016) 285-290. https://doi.org/10.1016/j.talanta.2015.08.052 
[15] N. Le Thao Nguyen, C.Y. Park, J.P. Park, S.K. Kailasa and T.J. Park, Synergistic molecular assembly of an aptamer and surfactant on gold nanoparticles for the colorimetric detection of trace levels of $\mathrm{As}^{3+}$ ions in real samples, New J. Chem. 42 (2018) 11530-11538. https://doi.org/10.1039/C8NJ01097H

[16] K. Vega-Figueroa, J. Santillán, V. Ortiz-Gómez, EO Ortiz-Quiles, BA Quiñones-Colón, Castilla- DA Casadiego, J Almodóvar, MJ Bayro, JA Rodríguez-Martínez, E. Nicolau, AptamerBased Impedimetric Assay of Arsenite in Water: Interfacial Properties and Performance, ACS Omega 3 (2018) 437-1444. https://doi.org/10.1021/acsomega.7b01710

[17] K. Matsunaga, Y. Okuyama, R. Hirano, S. Okabe, M. Takahashi, H. Satoh, Development of a simple analytical method to determine arsenite using a DNA aptamer and gold nanoparticles, Chemosphere 224 (2019) 538-543. https://doi.org/10.1016/j.chemosphere.2019.02.182

[18] Spuches AM, Kruszyna HG, Rich AM, Wilcox DE. Thermodynamics of the As(III)-thiol interaction: arsenite and monomethylarsenite complexes with glutathione, dihydrolipoic acid, and other thiol ligands. Inorg Chem. 2005 Apr 18;44(8):2964-72.

https://doi.org/10.1021/ic048694q

[19] Zong C, Zhang Z, Liu B, Liu J. Adsorption of Arsenite on Gold Nanoparticles Studied with DNA Oligonucleotide Probes. Langmuir. 2019 Jun 4;35(22):7304-7311.

https://doi.org/10.1021/acs.langmuir.9b01161

[20] Zong C, Liu J. The Arsenic-Binding Aptamer Cannot Bind Arsenic: Critical Evaluation of Aptamer Selection and Binding. Anal Chem. 2019 Aug 20;91(16):10887-10893.

https://doi.org/10.1021/acs.analchem.9b02789 